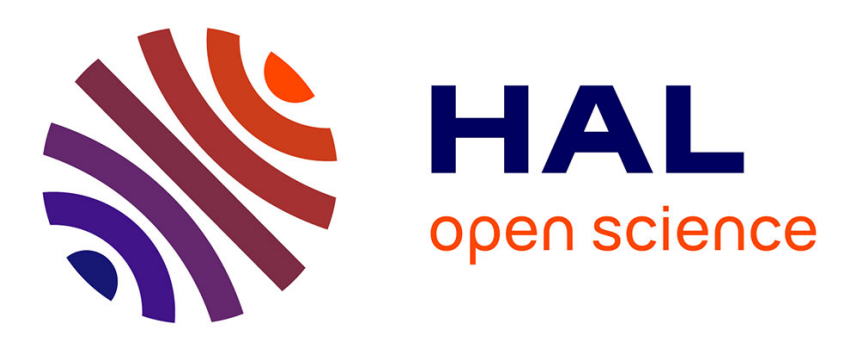

\title{
On some extremal problems for analytic functions with constraints on real or imaginary parts
}

\author{
Juliette Leblond, Dmitry Ponomarev
}

\section{To cite this version:}

Juliette Leblond, Dmitry Ponomarev. On some extremal problems for analytic functions with constraints on real or imaginary parts. Fabrizio Colombo; Irene Sabadini; Daniele C. Struppa; Mihaela B. Vajiac. Advances in Complex Analysis and Operator Theory: Festschrift in Honor of Daniel Alpay's 60th Birthday, Birkhauser, pp.219-236, 2017, 10.1007/978-3-319-62362-7_8 . hal-01623281

\section{HAL Id: hal-01623281 \\ https://hal.science/hal-01623281}

Submitted on 26 Oct 2017

HAL is a multi-disciplinary open access archive for the deposit and dissemination of scientific research documents, whether they are published or not. The documents may come from teaching and research institutions in France or abroad, or from public or private research centers.
L'archive ouverte pluridisciplinaire HAL, est destinée au dépôt et à la diffusion de documents scientifiques de niveau recherche, publiés ou non, émanant des établissements d'enseignement et de recherche français ou étrangers, des laboratoires publics ou privés. 


\title{
On some extremal problems for analytic func- tions with constraints on real or imaginary parts
}

\author{
Juliette Leblond and Dmitry Ponomarev
}

\begin{abstract}
We study some approximation problems by functions in the Hardy space $H^{2}$ of the upper half-plane or by their real or imaginary parts, with constraint on their real or imaginary parts on the boundary. Situations where the criterion acts on subsets of the boundary or of horizontal lines inside the half-plane are considered. Existence and uniqueness results are established, together with novel solution formulas and techniques. As a by-product, we devise a regularized inversion scheme for Poisson and conjugate Poisson integral transforms.
\end{abstract}

Mathematics Subject Classification (2000). 30E10, 30E20, 30E25, 45E10.

Keywords. Bounded extremal problems, constrained optimization, Hardy classes, Poisson and conjugate Poisson transforms, elliptic boundary value problems with incomplete data, regularization of ill-posed problems.

\section{Introduction}

Bounded extremal problems in Hardy classes of complex valued analytic functions have been widely considered in $[4,11,12,17]$ and the references therein and below. Being given a function on a subset $K$ of the boundary $\partial \Omega$ of a set $\Omega \subset \mathbb{C}$, they consist in best approximating that function on $K$ by a function in the Hardy space of $\Omega$, subject to some norm constraint on the complementary set $\partial \Omega \backslash K$ of the boundary where no data are available. Whenever $\Omega$ is the unit disk and $K=\partial \Omega$ is equal to its full boundary (the unit circle), they coincide with classical extremal problems, see [19, Ch. 8], [21, Ch. IV].

Such constrained best approximation problems arise in the context of system theory, for harmonic identification purposes and recovery of transfer functions from partial boundary data, see [13], in Hardy classes of the unit disk (for discrete time systems) or of the right-half plane (for continuous time). They also happen to furnish regularized resolution schemes for overdetermined boundary value problems 
concerning Laplace or elliptic partial differential equations in domains of dimension 2 , see $[16,23,27,30]$. In these frameworks, data are provided by approximate pointwise values (measurements, corrupted by errors) of a function belonging to some Hardy class or of its real or imaginary part, partially available on a subset of the domain or of its boundary. From these data, the aim is to recover the function itself.

Further, properties of Hardy spaces allow to establish stability results of such inverse problems, as in [15], and robustness properties of the approximation schemes, as in [22].

Here, we work in the Hilbertian setting and we consider situations where the domain $\Omega$ is the upper half-plane.

One typical example of such a problem is the approximation of a given function on a segment by traces of analytic functions controlling its behavior on the complement of the segment. Posed in a half-plane setting, the formulation of this problem reads as Problem 0 below.

Let $\Pi_{+}:=\{x+i y \in \mathbb{C}: \quad x \in \mathbb{R}, y>0\}$ be the upper half-plane and

$$
H_{+}^{2}:=H^{2}\left(\Pi_{+}\right):=\left\{g \text { analytic in } \Pi_{+}: \sup _{y>0} \int_{\mathbb{R}}|g(x+i y)|^{2} d x<\infty\right\}
$$

be the Hardy space of $\Pi_{+}\left[21, \mathrm{Ch}\right.$. II] which is similar to $H^{2}(\mathbb{D})$ (Hardy space for the unit disk) but not equivalent to it.

We will also identify functions $g \in H_{+}^{2}$ with their (non-tangential) boundary values $\lim _{y \rightarrow 0^{+}} g(\cdot+i y) \in L^{2}(\mathbb{R})$.

Given real numbers $-\infty<a_{k}<b_{k}<a_{k+1}<b_{k+1}<\infty, k=1, \ldots, N-1$, $N>1$, let $K:=\bigcup_{k=1}^{N}\left(a_{k}, b_{k}\right) \subset \mathbb{R}$ and $J:=\mathbb{R} \backslash K$. For the sake of explicitness of constructive aspects, without loss of generality, we simply assume that $K=(a, b)$.

\section{Problem 0}

Given functions $f_{0} \in L^{2}(K), h_{0} \in L^{2}(J)$, and a constant $M_{0}>0$, find $g_{0}$ such that

$$
g_{0}=\arg \min _{g \in H_{+}^{2},\left\|g-h_{0}\right\|_{L^{2}(J)} \leq M_{0}}\left\|g-f_{0}\right\|_{L^{2}(K)} .
$$

In this paper, we focus on two particular instances of bounded extremal problems involving approximation and constraints on real or imaginary parts, Problems 1 and 2, also mentioning some relevant problems.

\section{Problem 1}

Given a function $f_{1} \in L^{2}(K)$, a real-valued function $h_{1} \in L_{\mathbb{R}}^{2}(J)$, and a constant $M_{1} \geq 0$, find $g_{1}$ such that

$$
g_{1}=\arg \min _{g \in H_{+}^{2},\left\|\operatorname{Im} g-h_{1}\right\|_{L^{2}(J)} \leq M_{1}}\left\|g-f_{1}\right\|_{L^{2}(K)} .
$$


In these two problems, both the criterion and the constraint act on the boundary values of $H_{+}^{2}$ functions. This is not the case of Problem 2 below, where the constraint applies on the boundary values while the criterion applies to the function on some subset of the upper half-plane.

Let $S:=\bigcup_{k=1}^{N}\left(a_{k}, b_{k}\right) \subset \mathbb{R}$, for $-\infty<a_{k}<b_{k}<a_{k+1}<b_{k+1}<\infty, k=$ $1, \ldots, N-1, N>1$.

In what follows, we will write "supp" for the support of a function.

\section{Problem 2}

Given a real-valued functions $f_{2}, h_{2} \in L_{\mathbb{R}}^{2}(S)$ and constants $M_{2}, y_{0}>0$, find $g_{2} \in \mathcal{B}_{2} \subset H_{+}^{2}$ such that

$$
g_{2}=\arg \min _{g \in \mathcal{B}_{2}}\left\|\operatorname{Re} g\left(\cdot+i y_{0}\right)-f_{2}\right\|_{L^{2}(S)},
$$

where

$$
\begin{gathered}
\mathcal{B}_{2}:=\left\{g \in H_{+}^{2}: \operatorname{supp} \lim _{y \rightarrow 0^{+}} \operatorname{Re} g(\cdot+i y) \subset S,\left\|\lim _{y \rightarrow 0^{+}} \operatorname{Re} g(\cdot+i y)-h_{2}\right\|_{L^{2}(S)} \leq M_{2}\right\} \\
=\left\{g \in H_{+}^{2}: \quad \operatorname{supp} \operatorname{Re} g \subset S,\left\|\operatorname{Re} g-h_{2}\right\|_{L^{2}(S)} \leq M_{2}\right\} .
\end{gathered}
$$

Problem 1 (precisely, its version on a unit disk) was introduced in [23] where it has been qualitatively analyzed, and its solution has been implicitly characterized in terms of Toeplitz and Hankel operators, see also [27]. An approximate procedure based on numerical inversion of finite Toeplitz/Hankel matrices has also been proposed. As it was noted there, this problem reduces to Problem 0 whose solution can be explicitly (up to solving some trancendental equation for the value of a Lagrange parameter) written in an integral form generalizing in some sense a well-known Carleman formula $[1,26,31,34]$ for the case when $h_{0} \neq 0$. The obtained implicit characterization of the solution then reduces to an integral equation on $J$ with a symmetric kernel which is smooth in the interior of $J$ and bounded at the endpoints.

Problem 2 arises as a toy (two-dimensional) version of some physical inverse problems. More precisely, in Earth and planetary sciences, for study in paleomagnetism, an important inverse problem is to recover the magnetization contained in thin rock samples from partial measurements of their weak magnetic field, specifically of its normal component, taken on a planar set parallel to and at some distance of the sample, see [10, 29]. This is actually a three-dimensional issue, that involve both the Poisson kernel of the upper half-space and its conjugate, and their truncations to planar subsets (rectangles) of the upper half-space or its boundary.

Even though it has a real part constraint, Problem 2 is of a different type than Problems 0 and 1 as the approximation is performed on a subset of the analyticity domain rather than on a part of its boundary (see also Section 3.4). 
We show that solution of Problem 2 can be obtained from solution of an auxiliary integral equation with a simple (Poisson) kernel. Particular cases of the latter have been studied over the years. Recently, asymptotic resolution schemes have been developed for the case when $S$ is a single interval [36], depending on whether the ratio $|S| / y_{0}$ is large or small. Solutions are obtained as the eigenfunctions of the truncated Poisson operator and furnish a suitable basis for computation of solutions to Problem 2.

Problem 2 and its variation Problem $2^{\prime \prime}$ (introduced in Section 3.4) can also be viewed as regularized inversion settings for Poisson and conjugate Poisson transforms [35, 38] on $L^{2}(S)$.

In Section 2, we recall the properties of $H_{+}^{2}$ that we will need to establish existence and uniqueness of solutions to Problems 0, 1, 2 in Section 3. In Section 4, we obtain explicit constructive forms of the solutions. Some concluding comments are given in Section 5 .

\section{Properties of Hardy spaces}

We list below the basic properties of $H_{+}^{2}$ that will be used for the study of Problems 0, 1, 2, see [2, Sec. 8.6], [19, Ch. 11], [21, Ch. II, III], [24, Ch. 8], [25, Ch. VI].

- Functions in $H_{+}^{2}$ possess $L^{2}(\mathbb{R})$ boundary values that form a closed subspace of $L^{2}(\mathbb{R})$ and thus also form a Hilbert space, with the inner product

$$
\langle f, g\rangle_{L^{2}}=\int_{\mathbb{R}} f(x) \overline{g(x)} d x .
$$

- Functions in $H_{+}^{2}$ are uniquely determined by their boundary values on subsets of $\mathbb{R}$ of positive measure [21, Ch. II, Cor. 4.2], or [24, Ch. 8] (F. and M. Riesz theorem).

- $L^{2}(\mathbb{R})=H_{+}^{2} \oplus H_{-}^{2}$ (identified with their boundary values) with $H_{-}^{2}:=H^{2}\left(\Pi_{-}\right):=\left\{g\right.$ analytic in $\left.\Pi_{-}: \sup _{y<0} \int_{\mathbb{R}}|g(x+i y)|^{2} d x<\infty\right\}$

being the Hardy space of the lower half-plane

$$
\Pi_{-}:=\{x+i y \in \mathbb{C}: \quad x \in \mathbb{R}, y<0\},
$$

which can be established using Poisson and Cauchy representation formula [21, Ch. II, Sec. 3], [24, Ch. 8], [25, Ch. VI] (or from Paley-Wiener theorem [37, Ch. 2]).

For $y>0$, let us define the Hilbert transform, the Poisson and conjugate Poisson transforms $\mathcal{H}, P_{y}, Q_{y}$, respectively, as operators $L^{2}(\mathbb{R}) \rightarrow L^{2}(\mathbb{R})$ by, see $[21$, Ch. I, III]:

$$
\mathcal{H}[f](x):=\frac{1}{\pi} f_{\mathbb{R}} \frac{f(t)}{x-t} d t,
$$




$$
P_{y}[f](x):=\frac{y}{\pi} \int_{\mathbb{R}} \frac{f(t) d t}{(x-t)^{2}+y^{2}}, \quad Q_{y}[f](x):=\frac{1}{\pi} \int_{\mathbb{R}} \frac{(x-t) f(t)}{(x-t)^{2}+y^{2}} d t .
$$

The Hilbert transform $\mathcal{H}$ (or the harmonic conjugation operator) is bounded and isometric in $L^{2}(\mathbb{R})$ (see also Section 3 ). The projection operators $P_{ \pm}$from $L^{2}(\mathbb{R})$ onto $H_{ \pm}^{2}$ can be written as

$$
\begin{aligned}
P_{ \pm}[f](x) & =\lim _{y \rightarrow 0^{+}}\left(P_{ \pm y}+i Q_{ \pm y}\right)[f](x)= \pm \frac{1}{2} f(x)+\frac{i}{2} \mathcal{H}[f](x) \\
& = \pm \frac{1}{2} f(x)+\frac{1}{2 \pi i} f_{\mathbb{R}} \frac{f(t) d t}{t-x}
\end{aligned}
$$

which can also be seen as Plemelj-Sokhotskii formulas for $L^{2}$ functions ([20, Ch. 1] and [32, Ch. 2]).

The following density and unstability properties of Hardy functions provide motivation for bounded extremal problems [28, Lem. 2.1, Prop. 2.2]. Let $U \subset \mathbb{R}$, such that $|U|>0$ and $|\mathbb{R} \backslash U|>0$.

- The restrictions to $U$ of boundary values of $H_{+}^{2}$ functions form a dense subset of $L^{2}(U)$.

- Let $f \in L^{2}(U)$ and $g_{n} \in H_{+}^{2}$ such that $\left\|f-g_{n}\right\|_{L^{2}(U)} \rightarrow 0$; then, either $f$ coincides with the restriction to $U$ of some $H_{+}^{2}$ function, or $\left\|g_{n}\right\|_{L^{2}(\mathbb{R} \backslash U)} \rightarrow \infty$ as $n \rightarrow \infty$.

Note that similar properties for Hardy spaces $H^{p}(\mathbb{D})$ of the unit disk were also established and used in $[4,11,12]$ in order to study bounded extremal problems similar to Problem 0.

Before passing to the next section, let us adopt a few conventions. We will denote the characteristic function of any subset $U \subset \mathbb{R}$ as $\chi_{U}$. We will also use concatenation to construct a function from two complementary subsets of the line, namely, we write $f \vee h$ to mean a function which equals $f$ on $U \subset \mathbb{R}$ and $h$ on $V:=\mathbb{R} \backslash U$. For simplicity, we will put $\chi_{U} u=u \vee 0$ on $\mathbb{R}$ even if $u$ is defined on $U$ only.

\section{Analysis of the problems}

We are going to show that the problems formulated above admit a unique solution which also saturates the constraint under additional assumptions, and mention some variations of these problems.

\subsection{Preliminaries}

We will need the following results established in [17, Sec. 2] for complex Hilbert spaces which remain also true for real Hilbert spaces as only real-linear arguments were used in the proofs. 
Let $\mathcal{X}, \mathcal{K}, \mathcal{J}$ be Hilbert spaces and $A: \mathcal{X} \rightarrow \mathcal{K}, B: \mathcal{X} \rightarrow \mathcal{J}$ be bounded linear operators such that there exists a constant $\eta>0$,

$$
\|A g\|_{\mathcal{K}}^{2}+\|B g\|_{\mathcal{J}}^{2} \geq \eta\|g\|_{\mathcal{X}}^{2}, \forall g \in \mathcal{X}
$$

Assume that $A$ and $B$ have dense ranges and, for $f \in \mathcal{K}, h \in \mathcal{J}, M>0$, consider the nonempty closed convex set:

$$
\mathcal{B}=\mathcal{B}(h, M):=\left\{g \in \mathcal{X}: \quad\|B g-h\|_{\mathcal{J}} \leq M\right\} .
$$

We will write $A^{\star}$ and $B^{\star}$ for the adjoint operators to $A$ and $B$, respectively.

Lemma 1. [17, Lem. 2.1] Let $f \in \mathcal{K}, h \in \mathcal{J}$, and $M>0$. There exists a solution $g_{\star} \in \mathcal{X}$ to the bounded extremal problem:

$$
g_{\star}=\arg \min _{g \in \mathcal{B}}\|A g-f\|_{\mathcal{K}}
$$

Moreover, if $f \notin A(\mathcal{B})$, the solution $g_{\star}$ is unique and saturates the constraint: $\left\|B g_{\star}-h\right\|_{\mathcal{J}}=M$.

Lemma 2. [17, Thm 2.1] Let $f \in \mathcal{K} \backslash A(\mathcal{B}), h \in \mathcal{J}$, and $M>0$. The solution to the bounded extremal problem in Lemma 1 satisfies the operator equation

$$
\left(A^{\star} A+\gamma B^{\star} B\right) g_{\star}=A^{\star} f+\gamma B^{\star} h,
$$

where $\gamma>0$ is the unique constant such that $\left\|B g_{\star}-h\right\|_{\mathcal{J}}=M$.

Remark 3. Note that recovery situations where $f \in A(\mathcal{B})$ correspond to $A g_{\star}=f$, the preimage $g_{\star} \in \mathcal{B}$ of $f$ by $A$ being a solution that may not saturate the constraint (in this case, $\gamma=0$ in equation (3.2)). Uniqueness of $g_{\star}$ in this case holds if, and only if, $\left.A\right|_{\mathcal{B}}$ is injective.

We observe that we can view the complex-linear space $H_{+}^{2}$ as a real-linear Hilbert space with the inner product $\langle f, g\rangle_{L_{\mathbb{R}}^{2}}=\operatorname{Re}\langle f, g\rangle_{L^{2}}$, where $\langle f, g\rangle_{L^{2}}$ is as in (2.1) for $f, g \in H_{+}^{2}$. Indeed, because harmonic conjugation is realized by the Hilbert transform operator $\mathcal{H}$, the Hardy space $H_{+}^{2}$ can be represented as $(1+i \mathcal{H}) L^{2}(\mathbb{R})$. The latter complex-linear space is isomorphic to the real-linear space $(1+i \mathcal{H}) L_{\mathbb{R}}^{2}(\mathbb{R})$. To see this, take an arbitrary function $F=\operatorname{Re} F+i \operatorname{Im} F \in$ $L^{2}(\mathbb{R})$, and using the operator identity $\mathcal{H} \mathcal{H}=-1$, we proceed as

$$
\begin{aligned}
(1+i \mathcal{H}) F & =(1+i \mathcal{H}) \operatorname{Re} F+(i-\mathcal{H}) \operatorname{Im} F=(1+i \mathcal{H}) \operatorname{Re} F-\mathcal{H}(1+i \mathcal{H}) \operatorname{Im} F \\
& =(1+i \mathcal{H})(\operatorname{Re} F-\mathcal{H}[\operatorname{Im} F]) .
\end{aligned}
$$

Now, if we decompose $F=F_{+}+F_{-}$with $F_{ \pm} \in H_{ \pm}^{2}$, we note that $\operatorname{Re} F_{+}=$ $-\mathcal{H}\left[\operatorname{Im} F_{+}\right]$and $\operatorname{Re} F_{-}=\mathcal{H}\left[\operatorname{Im} F_{-}\right]$, and therefore,

$$
(1+i \mathcal{H}) F=2(1+i \mathcal{H}) \operatorname{Re} F_{+} .
$$

We thus established that $H_{+}^{2}$ is isomorphic to the real-linear space $(1+i \mathcal{H}) L_{\mathbb{R}}^{2}(\mathbb{R})$ which is further isomorphic to the real vector-function space $H_{+}^{2}:=(1, \mathcal{H})^{T} L_{\mathbb{R}}^{2}(\mathbb{R}) \subset$ 
$L_{\mathbb{R}}^{2}(\mathbb{R}) \times L_{\mathbb{R}}^{2}(\mathbb{R})$ with the inner product

$$
\begin{array}{r}
\left(w_{1}, w_{2}\right)_{L_{\mathbb{R}}^{2}}=\left\langle u_{1}, u_{2}\right\rangle_{L^{2}}+\left\langle\mathcal{H} u_{1}, \mathcal{H} u_{2}\right\rangle_{L^{2}}=2\left\langle u_{1}, u_{2}\right\rangle_{L^{2}}, \\
w_{k}=\left(u_{k}, \mathcal{H} u_{k}\right)^{T} \in \mathrm{H}_{+}^{2}, \quad u_{k} \in L_{\mathbb{R}}^{2}(\mathbb{R}), \quad k=1,2,
\end{array}
$$

where the isometric property of the Hilbert transform was taken into account. On the other hand, by the same property, we have, for $f, g \in H_{+}^{2}$,

$$
\langle f, g\rangle_{L_{\mathbb{R}}^{2}}=\operatorname{Re}\langle f, g\rangle_{L^{2}}=\langle\operatorname{Re} f, \operatorname{Re} g\rangle_{L^{2}}+\langle\operatorname{Im} f, \operatorname{Im} g\rangle_{L^{2}}=2\langle\operatorname{Re} f, \operatorname{Re} g\rangle_{L^{2}},
$$

which, upon identification $u_{1} \leftrightarrow \operatorname{Re} f, u_{2} \leftrightarrow \operatorname{Re} g$, justifies the use of the real inner product.

\subsection{Problems 0 and 1: solution existence and uniqueness}

We establish existence and uniqueness properties for solutions to Problems 0 and 1, beginning with Problem 0.

Theorem 4. Problem 0 admits a unique solution $g_{0}$. Moreover, if

$$
\left.f_{0} \notin\left\{g \in H_{+}^{2},\left\|g-h_{0}\right\|_{L^{2}(J)} \leq M_{0}\right\}\right|_{K},
$$

then it saturates the constraint: $\left\|g_{0}-h_{0}\right\|_{L^{2}(J)}=M_{0}$.

Proof. Here, we consider $H_{+}^{2}$ as a Hilbert space with the complex inner product (2.1) and define the bounded linear operators $A_{0}: H_{+}^{2} \rightarrow L^{2}(K), B_{0}: H_{+}^{2} \rightarrow$ $L^{2}(J)$ as restrictions to $K$ and $J$, respectively, of $H_{+}^{2}$ functions. Clearly, both $A_{0}$ and $B_{0}$ are bounded linear operators, and because of the density property of restrictions of boundary values of $H_{+}^{2}$ functions (recall Section 2), we see that these operators have dense ranges and that the approximation set is not empty. Also, since $K$ and $J$ are complementary sets, we have, for all $g \in H_{+}^{2}$,

$$
\left\|A_{0} g\right\|_{L^{2}(K)}^{2}+\left\|B_{0} g\right\|_{L^{2}(J)}^{2}=\|g\|_{L^{2}(\mathbb{R})}^{2},
$$

and hence (3.1) holds true with $\eta=1$. Therefore, Lemma 1 implies existence of the solution which, by Lemma 2 , is unique and saturates the constraint if $f_{0} \notin$ $\left.\left\{g \in H_{+}^{2},\left\|g-h_{0}\right\|_{L^{2}(J)} \leq M_{0}\right\}\right|_{K}$.

Finally, if $\left.f_{0} \in\left\{g \in H_{+}^{2},\left\|g-h_{0}\right\|_{L^{2}(J)} \leq M_{0}\right\}\right|_{K}$, Remark 3 is to the effect that uniqueness still holds. Indeed, $A_{0}$ is injective on $H_{+}^{2}$, because $H_{+}^{2}$ functions cannot vanish on the set $K$ of positive Lebesgue measure unless they identically vanish.

For the above proof, observe that we could also proceed as in [37] using [28] and the orthogonal decomposition $L^{2}(\mathbb{R})=H_{+}^{2} \oplus H_{-}^{2}$.

We now turn to Problem 1. 
Theorem 5. Problem 1 admits a unique solution $g_{1}$. Moreover, if

$$
\left.f_{1} \notin\left\{g \in H_{+}^{2},\left\|I m g-h_{1}\right\|_{L^{2}(J)} \leq M_{1}\right\}\right|_{K},
$$

then it saturates the constraint: $\left\|I m g_{1}-h_{1}\right\|_{L^{2}(J)}=M_{1}$.

Proof. Considering the equivalent real Hilbertian structure for $H_{+}^{2}$ discussed in Section 3.1, we can prove Theorem 5 using Lemmas 1 and 2. Let the operator $A_{1}=A_{0}$ be as in the proof of Theorem 4 and $B_{1}: H_{+}^{2} \rightarrow L_{\mathbb{R}}^{2}(J)$ be the imaginary part of the restriction to $J$ of $H_{+}^{2}$ functions, which, clearly, is also a bounded linear operator. As for Problem 0, $A_{1}$ has dense range property, so is the case for $B_{1}$ (in fact, $B_{1}$ is surjective: for any $h \in L_{\mathbb{R}}^{2}(J)$, we can construct the function $\left.\left(-\mathcal{H}\left[\chi_{J} h\right]+i\left(\chi_{J} h\right)\right) \in H_{+}^{2}\right)$. Further, the approximation set is not empty and property (3.1) holds for all $g \in H_{+}^{2}$ with $B=B_{1}$. Indeed, for $g \in H_{+}^{2}$, because $\operatorname{Im} g$ is the conjugate function of $\operatorname{Re} g$ and Hilbert transform is an isometry on $L^{2}(\mathbb{R})$, we get

$$
\begin{aligned}
\left\|A_{1} g\right\|_{L^{2}(K)}^{2}+\left\|B_{1} g\right\|_{L^{2}(J)}^{2} & =\|g\|_{L^{2}(K)}^{2}+\|\operatorname{Im} g\|_{L^{2}(J)}^{2} \\
& =\|\operatorname{Re} g\|_{L^{2}(K)}^{2}+\|\operatorname{Im} g\|_{L^{2}(\mathbb{R})}^{2} \geq \frac{1}{2}\|g\|_{L^{2}(\mathbb{R})}^{2},
\end{aligned}
$$

whence (3.1) holds true with $\eta=\frac{1}{2}$. If $\left.f_{1} \notin\left\{g \in H_{+}^{2},\left\|\operatorname{Im} g-h_{1}\right\|_{L^{2}(J)} \leq M_{1}\right\}\right|_{K}$, then Lemmas 1, 2 again directly lead to the results.

If $\left.f_{1} \in\left\{g \in H_{+}^{2},\left\|\operatorname{Im} g-h_{1}\right\|_{L^{2}(J)} \leq M_{1}\right\}\right|_{K}$, we argue as at the end of the proof of Theorem 4 using Remark 3.

For proving Theorems 4, 5, other possibilities consist in using best approximation projections on closed convex subsets of a Hilbert space as in [23], or weak-compactness arguments as in [11].

\subsection{Problem 2: solution existence and uniqueness}

We now consider Problem 2.

Theorem 6. Problem 2 admits a unique solution $g_{2}$, i.e. there exists a unique function $g_{2} \in \mathcal{B}_{2} \subset H_{+}^{2}$ such that

$$
g_{2}=\arg \min _{g \in \mathcal{B}_{2}}\left\|\operatorname{Reg}\left(\cdot+i y_{0}\right)-f_{2}\right\|_{L^{2}(S)} \cdot
$$

Moreover, if $\left.f_{2} \notin R e \mathcal{B}_{2}\right|_{S \times\left\{y_{0}\right\}}$, then $g_{2}$ saturates the constraint: $\left\|\operatorname{Re} g_{2}-h_{2}\right\|_{L^{2}(S)}=$ $M_{2}$.

Proof. As in the proof of Theorem 5, we view $H_{+}^{2}$ as a real-linear Hilbert space with the inner product $\langle f, g\rangle_{L_{\mathbb{R}}^{2}}=\operatorname{Re}\langle f, g\rangle_{L^{2}}$ for $f, g \in H_{+}^{2}$. Consider the subspace

$$
\mathcal{T}:=\left\{g \in H_{+}^{2}: \quad \operatorname{supp} \operatorname{Re} g \subset S\right\} \subset H_{+}^{2},
$$

which is closed, and hence is a Hilbert space on its own account. 
Define the bounded linear operators $A_{2}: \mathcal{T} \rightarrow L_{\mathbb{R}}^{2}(S), B_{2}: \mathcal{T} \rightarrow L_{\mathbb{R}}^{2}(S)$ as

$$
\begin{aligned}
& A_{2} g(\cdot)=\operatorname{Re} g\left(\cdot+i y_{0}\right)=P_{y_{0}}[\operatorname{Re} g](\cdot) \quad \text { and } \\
& B_{2} g(\cdot)=\lim _{y \rightarrow 0^{+}} \operatorname{Re} g(\cdot+i y)=\operatorname{Re} g(\cdot) \quad \text { on } S .
\end{aligned}
$$

Note that $B_{2}$ is surjective, as discussed in the proof of Theorem 5 . To verify that $A_{2}$ has a dense range, it is enough to show that it is the case for the same map when its domain of definition is $L_{\mathbb{R}}^{2}(S)$. The latter is an operator still given by $P_{y_{0}}$ which is now a self-adjoint operator (as a convolution operator with an even kernel) on $L_{\mathbb{R}}^{2}(S)$. Hence, to show the density of its range, it is enough to check its injectivity. The injectivity can be deduced from the non-vanishing property of harmonic functions on non-empty open subsets of the domain of harmonicity $[6$, Thms $1.27,1.28]$ (note that $\operatorname{Re} \mathcal{B}_{2}$ consists of functions harmonic in $\Pi_{+}$). Combined with the density of $\left.\operatorname{Re} \mathcal{T}\right|_{S}$ in $L_{\mathbb{R}}^{2}(S)$ (given $f \in L_{\mathbb{R}}^{2}(S)$, we construct $\left.\left(f \chi_{S}+i \mathcal{H}\left[f \chi_{S}\right]\right) \in \mathcal{T}\right)$, this leads to the dense range property of $A_{2}$. The fact that (3.1) is satisfied by $A_{2}$ and $B_{2}$ can easily be checked, for $g \in \mathcal{T}$,

$$
\left\|\operatorname{Re} g\left(\cdot+i y_{0}\right)\right\|_{L^{2}(S)}^{2}+\|\operatorname{Re} g\|_{L^{2}(S)}^{2} \geq\|\operatorname{Re} g\|_{L^{2}(S)}^{2}=\|\operatorname{Re} g\|_{L^{2}(\mathbb{R})}^{2}=\frac{1}{2}\|g\|_{L^{2}(\mathbb{R})}^{2},
$$

as in the proof of Theorem 5. Therefore, Lemmas 1 and 2 again furnish the proof whenever $\left.f_{2} \notin \operatorname{Re} \mathcal{B}_{2}\right|_{S \times\left\{y_{0}\right\}}$.

Finally, if $\left.f_{2} \in \operatorname{Re} \mathcal{B}_{2}\right|_{S \times\left\{y_{0}\right\}}$, thanks again to Remark $3, g_{2}$ is unique for $A_{2}$ is injective (as discussed in the previous paragraph).

Observe that in the present two-dimensional case, Theorems 4, 5 still hold whenever $K$ is a more general set of finite positive Lebesgue measure [4, 11, 23]. This should also be the case for Theorem 6 if $S$ is of finite positive Lebesgue measure, see [7].

\subsection{More about Problems 1 and 2}

Note that Problem 1 is equivalent to a similar one, Problem $1^{\prime}$, with the constraint acting on $\operatorname{Re} g$ (multiplying by $\pm i$ the function $f_{1}$ and the approximant $g$ ): given a function $f_{1} \in L^{2}(K)$, a real-valued function $h_{1} \in L_{\mathbb{R}}^{2}(J)$, and a constant $M_{1} \geq 0$, find $g_{1}^{\prime}$ such that

$$
g_{1}^{\prime}=\arg \min _{g \in H_{+}^{2},\left\|\operatorname{Re} g-h_{1}\right\|_{L^{2}(J)} \leq M_{1}}\left\|g-f_{1}\right\|_{L^{2}(K)} .
$$

In Problem 1, situations when $M_{1}=0$ could be analyzed as they correspond to the constraint $\operatorname{Im} g=h_{1}$ a.e. in $K$, or $\operatorname{Re} g=h_{1}$ if we discuss its Problem $1^{\prime}$ version. In this case, solutions $g_{1}^{\prime} \in H_{+}^{2}$ are such that on $\mathbb{R}, g_{1}^{\prime}=(1+i \mathcal{H})\left[\phi_{1} \vee h_{1}\right]$ for some $\phi_{1} \in L_{\mathbb{R}}^{2}(K)$, i.e. we look for $\phi_{1}$ such that $\phi_{1}=\arg \min _{\phi \in L_{\mathbb{R}}^{2}(K)}\left\|f_{1}-\phi-i \mathcal{H}\left[\phi \vee h_{1}\right]\right\|_{L^{2}(K)}$.

The above Problem $1^{\prime}$ is related to the following Problem 3 (see also [27, Prob. $2.2])$. Given real-valued functions $f_{3} \in L_{\mathbb{R}}^{2}(K), h_{3} \in L_{\mathbb{R}}^{2}(J)$, and a constant 
$M_{3} \geq 0$, find $g_{3}$ such that

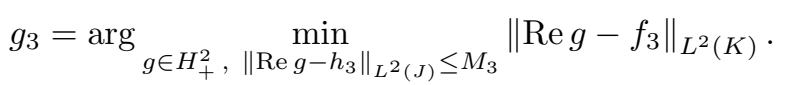

It is interesting to compare Problems 2 and 3 , and to point out the links and differences between their criteria and constraints. Indeed, with $S=J, h_{2}=h_{3}$, and $M_{2}=M_{3}$, the approximation set $\mathcal{B}_{2}$ in Problem 2 is a strict subset of the one in Problem 3, because of the additional constraint on the support of the real part of the approximant in Problem 2. A notable difference between them is that in Problem 2, the criterion acts on the subset $S \times\left\{y_{0}\right\}$ of $\Pi_{+}$while in Problem 3 it acts on the part $K=\mathbb{R} \backslash J$ of the boundary $\mathbb{R}$.

Concerning Problem 2, note that $\mathcal{T}=\left(P_{y}+i Q_{y}\right)\left[\chi_{S} u\right]$ for $u \in L_{\mathbb{R}}^{2}(S)$ (whence $\left.\mathcal{T}\right|_{\mathbb{R}}=(1+i \mathcal{H})\left[\chi_{S} u\right]$ ) and $\mathcal{B}_{2}=\left(P_{y}+i Q_{y}\right)\left[\chi_{S} u\right]$ for $u \in L_{\mathbb{R}}^{2}(S)$ such that $\left\|u-h_{2}\right\|_{L^{2}(S)} \leq M_{2}$. Then, Problem 2 rephrases as the one of finding $u_{2}$ solution to

$$
u_{2}=\arg \min _{u \in L_{\mathbb{R}}^{2}(S),\left\|u-h_{2}\right\|_{L^{2}(S)} \leq M_{2}}\left\|P_{y_{0}}\left[\chi_{S} u\right]-f_{2}\right\|_{L^{2}(S)},
$$

where only the restriction to $S$ of $P_{y_{0}}\left[\chi_{S} u\right]$ is involved in the above criterion. In particular, for $M_{2}=0$, this furnishes the unique solution $g_{2}=\left(P_{y}+i Q_{y}\right)\left[\chi_{S} h_{2}\right]$ to Problem 2 (which necessarily saturates the constraint and is equal to $(1+i \mathcal{H})\left[\chi_{S} h_{2}\right]$ on $\mathbb{R}$ ). For $M_{2}>0$, we can see that $\left.f_{2} \in \operatorname{Re} \mathcal{B}_{2}\right|_{S \times\left\{y_{0}\right\}}$ if and only if $f_{2}=P_{y_{0}}\left[\chi_{S} u\right]$ on $S$, for some $u \in L_{\mathbb{R}}^{2}(S)$ such that $\left\|u-h_{2}\right\|_{L^{2}(S)} \leq M_{2}$. This reformulation shows that Problem 2 is, in fact, nothing but a regularization scheme for inversion of Poisson transform [35] on $L^{2}(S)$.

This also furnishes another equivalent setting for establishing well-posedness of Problem 2, yet appropriate for Lemmas 1, 2. One can also make use of [14, Thm $5.2]$ in order to show that there exists $\gamma>0$ such that

$$
u_{2}=\arg \min _{u \in L_{\mathbb{R}}^{2}(S)}\left(\left\|P_{y_{0}}\left[\chi_{S} u\right]-f_{2}\right\|_{L^{2}(S)}^{2}+\gamma\left\|u-h_{2}\right\|_{L^{2}(S)}^{2}\right) .
$$

The saturation of the constraint then implies by differentiation that

$$
\left\langle P_{y_{0}}\left[\chi_{S} u_{2}\right]-f_{2}, P_{y_{0}}\left[\chi_{S} \varphi\right]\right\rangle_{L^{2}(S)}+\gamma\left\langle u_{2}-h_{2}, \varphi\right\rangle_{L^{2}(S)}=0, \forall \varphi \in L^{2}(S),
$$

for $\gamma$ such that $\left\|u_{2}-h_{2}\right\|_{L^{2}(S)}=M_{2}$.

We also note that Problem 2 is equivalent to the following Problem $2^{\prime}$ (multiplying by $\pm i$ the functions $f_{2}, h_{2}$ and the approximant $g$ ): given real-valued functions $f_{2}, h_{2} \in L_{\mathbb{R}}^{2}(S)$, find $g_{2}^{\prime}$ such that

$$
g_{2}^{\prime}=\arg \min _{g \in \mathcal{B}_{2}^{\prime}}\left\|\operatorname{Im} g\left(\cdot+i y_{0}\right)-f_{2}\right\|_{L^{2}(S)},
$$

where

$$
\mathcal{B}_{2}^{\prime}:=\left\{g \in H_{+}^{2}: \quad \operatorname{supp} \operatorname{Im} g \subset S,\left\|\operatorname{Im} g-h_{2}\right\|_{L^{2}(S)} \leq M_{2}\right\}
$$


Another interesting variation of Problem 2 involves both real and imaginary parts. Namely, we introduce Problem $2^{\prime \prime}$ : given real-valued functions $f_{2}, h_{2} \in L_{\mathbb{R}}^{2}(S)$, and the set $\mathcal{B}_{2}$ as in Problem 2, find $g_{2}^{\prime \prime}$ such that

$$
g_{2}^{\prime \prime}=\arg \min _{g \in \mathcal{B}_{2}}\left\|\operatorname{Im} g\left(\cdot+i y_{0}\right)-f_{2}\right\|_{L^{2}(S)} .
$$

The only difference in the treatment of this problem with respect to Problem 2 is the replacement of $A_{2}$ by the bounded linear operator $A_{2}^{\prime \prime}: \mathcal{T} \rightarrow L_{\mathbb{R}}^{2}(S)$ acting as

$$
A_{2}^{\prime \prime} g(\cdot)=\operatorname{Im} g\left(\cdot+i y_{0}\right)=Q_{y_{0}}[\operatorname{Re} g](\cdot) \text { on } S .
$$

In order to deduce existence and uniqueness of the solution, we only have to verify the range density property (the proof that (3.1) is satisfied is exactly the same as for Problem 2). Since $Q_{y_{0}} \chi_{S}=\mathcal{H} P_{y_{0}} \chi_{S}$, it follows directly from the facts that the range of $P_{y_{0}} \chi_{S}$ is dense in $L_{\mathbb{R}}^{2}(S)$ and that $\mathcal{H}$ is surjective.

\section{Computation of solutions}

\subsection{Problem 0}

A version of this problem for the unit disk has been considered in [4]. In [11], its solution has been obtained in an explicit integral form in terms of the socalled quenching function arising from the Goluzin-Krylov method (a Carleman formula) for the recovery of analytic functions from their partial boundary values $[1,26,31,34]$. We obtain an analogous integral formula using reduction to a Riemann-Hilbert problem [20, 32], which further allows us to treat Problem 1.

In order to make use of Lemma 2 for the characterization of the solution, we compute the adjoint operators $A_{0}^{\star}: L^{2}(K) \rightarrow H_{+}^{2}, B_{0}^{\star}: L^{2}(J) \rightarrow H_{+}^{2}$. Letting $g \in H_{+}^{2}, \phi \in L^{2}(K)$, we have

$$
\left\langle A_{0} g, \phi\right\rangle_{L^{2}(K)}=\left\langle A_{0} g, \chi_{K} \phi\right\rangle_{L^{2}}=\left\langle g, P_{+} \chi_{K} \phi\right\rangle_{L^{2}},
$$

yielding $A_{0}^{\star}=P_{+} \chi_{K}$, and similarly, $B_{0}^{\star}=P_{+} \chi_{J}$.

Equation (3.2) then leads to the critical point equation that must be satisfied by the solution $g_{0}$ to Problem 0:

$$
P_{+}\left[\left(1+\left(\gamma_{0}-1\right) \chi_{J}\right) g_{0}\right]=P_{+}\left(f_{0} \vee \gamma_{0} h_{0}\right),
$$

where the parameter $\gamma_{0}>0$ is chosen such that $\left\|g_{0}-h_{0}\right\|_{L^{2}(K)}=M_{0}$.

Equation (4.1) can be rewritten as

$$
\left(1+\left(\gamma_{0}-1\right) \chi_{J}\right) g_{0}=\left(f_{0} \vee \gamma_{0} h_{0}\right)+\psi
$$

with some unknown function $\psi \in H_{-}^{2}$. Equivalently,

$$
\gamma_{0} g_{0}=G \psi+L,
$$

where $G \in L^{\infty}(\mathbb{R})$ and $L \in L^{2}(\mathbb{R})$ are defined by:

$$
G(x):=\frac{\gamma_{0}}{1+\left(\gamma_{0}-1\right) \chi_{J}(x)}= \begin{cases}1, & x \in J \\ \gamma_{0}, & x \in K\end{cases}
$$




$$
L(x):=G(x)\left(f_{0} \vee \gamma_{0} h_{0}\right)(x)=\gamma_{0}\left(f_{0} \vee h_{0}\right)(x) .
$$

Since $\gamma_{0} g_{0} \in H_{+}^{2}$ and $\psi \in H_{-}^{2}$, equation (4.2) is a Riemann-Hilbert problem.

The standard resolution procedure (see, for instance, [20, Ch. 2] or [32, Ch. 5]) requires factorizing the coefficient of the problem $G$ as $G=G_{+} / G_{-}$ with zero-free functions $G_{+}$and $G_{-}$analytic in the upper and lower half-planes, respectively. To construct such a factorization, we consider the decomposition $\log G=\log G_{+}-\log G_{-}$which can be achieved by Plemelj-Sokhotskii formulas applied to the Cauchy integral of $\log G$. Due to its vanishing at infinity and to the positivity of $G$, this integral is well-defined, except at the endpoints $x=a, b$ of $K$ where it is merely bounded. Indeed,

$$
\begin{aligned}
\log G_{ \pm}(x) & =\lim _{y \rightarrow 0^{ \pm}} \int_{\mathbb{R}} \frac{\log G(t)}{t-(x+i y)} d t= \pm \frac{1}{2} \log \gamma_{0} \chi_{K}(x)+\frac{\log \gamma_{0}}{2 \pi i} f_{K} \frac{d t}{t-x} \\
& = \begin{cases}\frac{1}{2 \pi i} \log \gamma_{0} \log \left(\frac{x-a}{x-b}\right), & x \in J, \\
\pm \frac{1}{2} \log \gamma_{0}+\frac{1}{2 \pi i} \log \gamma_{0} \log \left(\frac{a-x}{x-b}\right), & x \in K,\end{cases}
\end{aligned}
$$

where the branches of logarithms in both expressions are real-valued on $J$ and $K$, respectively. Therefore,

$$
G_{ \pm}(x)=e^{\log G_{ \pm}(x)}= \begin{cases}\left(\frac{x-a}{x-b}\right)^{\frac{1}{2 \pi i} \log \gamma_{0}}, & x \in J, \\ \pm \sqrt{\gamma_{0}}\left(\frac{a-x}{x-b}\right)^{\frac{1}{2 \pi i} \log \gamma_{0}}, & x \in K,\end{cases}
$$

are the boundary values on $\mathbb{R}$, from above and below, of the Cauchy integral

$$
G(z)=e^{\log G(z)}=\exp \left[\frac{1}{2 \pi i} \int_{\mathbb{R}} \frac{\log G(t) d t}{t-z}\right] .
$$

Equation (4.2) now becomes

$$
\gamma_{0} g_{0} / G_{+}-\psi / G_{-}=L / G_{+},
$$

which is a jump problem since in the left-hand side we have the difference of $H_{+}^{2}$ and $H_{-}^{2}$ functions and the right-hand side is in $L^{2}(\mathbb{R})$.

To solve this jump problem, we consider the Cauchy integral

$$
Y(z)=\frac{1}{2 \pi i} \int_{\mathbb{R}} \frac{L(t)}{G_{+}(t)} \frac{d t}{t-z}
$$

and, by Plemelj-Sokhotskii formulas

$$
Y_{ \pm}(x)= \pm \frac{1}{2} \frac{L(x)}{G_{+}(x)}+\frac{1}{2 \pi i} f_{\mathbb{R}} \frac{L(t)}{G_{+}(t)} \frac{d t}{t-x},
$$

we have, for $x \in \mathbb{R}, x \neq a, b$,

$$
L(x) / G_{+}(x)=Y_{+}(x)-Y_{-}(x) .
$$


This leads to

$$
\gamma_{0} g_{0} / G_{+}-Y_{+}=\psi / G_{-}-Y_{-},
$$

from which we deduce that both left- and right-hand sides are restrictions to $\mathbb{R}$ of one entire function. Since both $g_{0} / G_{+}$and $Y_{+}$decay at infinity in the upper halfplane, by Liouville theorem, we conclude that this entire function is identically zero. This entails that, for $x \in \mathbb{R}$,

$$
g_{0}(x)=\frac{1}{\gamma_{0}} G_{+}(x) Y_{+}(x)=\frac{1}{2 \gamma_{0}} L(x)+\frac{G_{+}(x)}{2 \gamma_{0} \pi i} f_{\mathbb{R}} \frac{L(t)}{G_{+}(t)} \frac{d t}{t-x},
$$

and, for $z \in \Pi_{+}$,

$$
g_{0}(z)=\frac{1}{\gamma_{0}} G(z) Y(z)=\frac{G(z)}{2 \gamma_{0} \pi i} \int_{\mathbb{R}} \frac{L(t)}{G_{+}(t)} \frac{d t}{t-z} .
$$

\subsection{Problem 1}

According to [23, Prop. 5 (i)], the solution $g_{1}$ to Problem 1 also solves Problem 0 with $f_{0}=f_{1}, h_{0}=i h_{1}+\operatorname{Re} g_{1}$ and $\gamma_{0}=\gamma_{1}>0$ chosen such that $\left\|\operatorname{Im} g_{1}-h_{1}\right\|_{L^{2}(J)}=M_{1}$.

Applying the above formulas (4.4)-(4.5), we arrive at

$$
\begin{gathered}
g_{1}(x)=\frac{1}{2} \operatorname{Re} g_{1}(x)+\frac{G_{+}(x)}{2 \pi i} f_{J} \frac{\operatorname{Re} g_{1}(t) d t}{G_{+}(t)(t-x)}+F_{0}(x), \quad x \in J, \\
g_{1}(x)=\frac{1}{2} f_{1}(x)+\frac{G_{+}(x)}{2 \pi i}\left[f_{K} \frac{f_{1}(t) d t}{G_{+}(t)(t-x)}+\int_{J} \frac{\left(\operatorname{Re} g_{1}(t)+i h_{1}(t)\right) d t}{G_{+}(t)(t-x)}\right], \quad x \in K \\
g_{1}(z)=\frac{G_{+}(z)}{2 \pi i}\left[\int_{K} \frac{f_{1}(t) d t}{G_{+}(t)(t-z)}+\int_{J} \frac{\left(\operatorname{Re} g_{1}(t)+i h_{1}(t)\right) d t}{G_{+}(t)(t-z)}\right], \quad z \in \Pi_{+}
\end{gathered}
$$

where

$F_{0}(x):=\frac{i}{2} h_{1}(x)+\frac{G_{+}(x)}{2 \pi} f_{J} \frac{h_{1}(t) d t}{G_{+}(t)(t-x)}+\frac{G_{+}(x)}{2 \pi i} \int_{K} \frac{f_{1}(t) d t}{G_{+}(t)(t-x)}, \quad x \in J$.

Observe that the solution expressions (4.7)-(4.8) will be complete once the involved values of $\operatorname{Re} g_{1}$ on $J$ are known. The latter can, in principle, be found from the solution of the integral equation obtained from (4.6) by taking real parts and using the fact that $\bar{G}_{+}=1 / G_{+}$

$$
\operatorname{Re} g_{1}(x)=\frac{1}{2 \pi i} \int_{J} \frac{\operatorname{Re} g_{1}(t)}{t-x}\left(\frac{G_{+}(x)}{G_{+}(t)}-\frac{G_{+}(t)}{G_{+}(x)}\right) d t+2 \operatorname{Re} F_{0}(x), \quad x \in J .
$$

Note that the integral equation for $\operatorname{Re} g_{1}$ has a regular symmetric kernel

$$
\mathcal{K}(x, t):=\frac{1}{x-t}\left(\frac{G_{+}(x)}{G_{+}(t)}-\frac{G_{+}(t)}{G_{+}(x)}\right)=\frac{G_{+}(x)-G_{+}(t)}{x-t}\left(\frac{G_{+}(x)+G_{+}(t)}{G_{+}(x) G_{+}(t)}\right),
$$

whose smoothness is seen from (4.3). Indeed, $G_{+}$is non-vanishing and smooth (except at the endpoints where it still remains bounded), which eliminates the 
zero of the denominator at $t=x$ in the first factor. This makes the obtained integral equation amenable to effective numerical resolution.

\subsection{Problem 2}

Let us compute the adjoint operators $A_{2}^{\star}: L_{\mathbb{R}}^{2}(S) \rightarrow \mathcal{T}, B_{2}^{\star}: L_{\mathbb{R}}^{2}(S) \rightarrow \mathcal{T}$.

For $g \in \mathcal{T}, \phi \in L_{\mathbb{R}}^{2}(S)$, using that $P_{y_{0}}$ is a self-adjoint operator (as a convolution integral operator with even kernel), we have:

$$
\begin{aligned}
\left\langle A_{2} g, \phi\right\rangle_{L^{2}(S)} & =\operatorname{Re}\left\langle P_{y_{0}} g, \chi_{S} \phi\right\rangle_{L^{2}}=\operatorname{Re}\left\langle g, P_{y_{0}} \chi_{S} \phi\right\rangle_{L^{2}}=\left\langle\operatorname{Re} g, P_{y_{0}} \chi_{S} \phi\right\rangle_{L^{2}(S)} \\
& =\operatorname{Re}\left\langle g, \chi_{S} P_{y_{0}} \chi_{S} \phi\right\rangle_{L^{2}}=\left\langle g, P_{+} \chi_{S} P_{y_{0}} \chi_{S} \phi\right\rangle_{L_{\mathbb{R}}^{2}}, \\
& \left\langle B_{2} g, \phi\right\rangle_{L^{2}(S)}=\operatorname{Re}\left\langle g, \chi_{S} \phi\right\rangle_{L^{2}}=\left\langle g, P_{+} \chi_{S} \phi\right\rangle_{L_{\mathbb{R}}^{2}},
\end{aligned}
$$

and hence $A_{2}^{\star}=P_{+} \chi_{S} P_{y_{0}} \chi_{S}, B_{2}^{\star}=P_{+} \chi_{S}$.

The characterization (3.2) of the solution $g_{2}$ now reads

$$
P_{+} \chi_{S} P_{y_{0}} \chi_{S} P_{y_{0}} \operatorname{Re} g_{2}+\gamma_{2} P_{+} \chi_{S} \operatorname{Re} g_{2}=P_{+}\left[\chi_{S} P_{y_{0}} \chi_{S} f_{2}+\gamma_{2} \chi_{S} h_{2}\right],
$$

where the parameter $\gamma_{2}>0$ is chosen such that $\left\|\operatorname{Re} g_{2}-h_{2}\right\|_{L^{2}(S)}=M_{2}$.

This implies

$$
\chi_{S} P_{y_{0}} \chi_{S} P_{y_{0}} \operatorname{Re} g_{2}+\gamma_{2} \chi_{S} \operatorname{Re} g_{2}=\chi_{S} P_{y_{0}} \chi_{S} f_{2}+\gamma_{2} \chi_{S} h_{2}+\psi,
$$

with some function $\psi \in H_{-}^{2}$. However, since all the terms in the equation vanish outside of the set $S$ and so must do $\psi$, but since $|\mathbb{R} \backslash S|>0$, this vanishing is prohibited by analyticity unless $\psi \equiv 0$ which is what we have to conclude. We thus arrive at the double integral equation on $S$

$$
\left[\left(P_{y_{0}} \chi_{S}\right)^{2}+\gamma_{2}\right] \operatorname{Re} g_{2}=P_{y_{0}} \chi_{S} f_{2}+\gamma_{2} h_{2}
$$

which can be solved, for example, in terms of eigenfunction expansion for the compact self-adjoint operator $\left.f \mapsto\left[P_{y_{0}} \chi_{S} f\right]\right|_{S}$ on $L_{\mathbb{R}}^{2}(S)$ considered in [36, Part II]. Indeed, this operator is compact (due to regularity of the kernel and boundedness of $S$ ) and self-adjoint, hence the spectral theorem for linear compact self-adjoint operators [33, Thm 6.11.1] implies the existence of a set of mutually orthogonal basis functions $\left(\phi_{n}\right)_{n=0}^{\infty}$ in $L_{\mathbb{R}}^{2}(S)$ and a set of numbers $\left(\lambda_{n}\right)_{n=0}^{\infty} \subset \mathbb{R}$ such that

$$
P_{y_{0}}\left[\chi_{S} \phi_{n}\right](x)=\frac{y_{0}}{\pi} \int_{S} \frac{\phi_{n}(t)}{(x-t)^{2}+y_{0}^{2}} d t=\lambda_{n} \phi_{n}(x), \quad x \in S .
$$

In [36, Part II], equation (4.10) was solved asymptotically when $S$ is a finite interval.

Expanding both the solution $\operatorname{Re} g_{2}$ and the right-hand side of (4.9) over eigenfunctions $\phi_{n}$ and assuming, without loss of generality, that their $L^{2}(S)$ norms are normalized to one, we conclude

$$
\operatorname{Re} g_{2}(x)=\sum_{n=0}^{\infty} \frac{1}{\lambda_{n}^{2}+\gamma_{2}}\left\langle P_{y_{0}} \chi_{S} f_{2}+\gamma_{2} h_{2}, \phi_{n}\right\rangle_{L^{2}(S)} \phi_{n}(x) .
$$


This allows to determine the parameter $\gamma_{2}$ from $\left\|\operatorname{Re} g_{2}-h_{2}\right\|_{L^{2}(S)}=M_{2}$, that can now be rewritten as (upon expansion of $h_{2}$ over $\phi_{n}$ ):

$$
\sum_{n=0}^{\infty}\left[\left\langle\frac{1}{\lambda_{n}^{2}+\gamma_{2}}\left(P_{y_{0}} \chi_{S} f_{2}+\gamma_{2} h_{2}\right)-h_{2}, \phi_{n}\right\rangle_{L^{2}(S)}\right]^{2}=M_{2}^{2}
$$

Then, for $z=x+i y \in \Pi_{+}$, the solution to Problem 2 is given by

$$
g_{2}(x+i y)=\frac{1}{2}\left(P_{y}+i Q_{y}\right)\left[\chi_{S} \operatorname{Re} g_{2}\right](x) .
$$

\subsection{Problem $2^{\prime \prime}$}

The resolution procedure can be repeated in the same way as for Problem 2 in Section 4.3; however, we now have $\left(A_{2}^{\prime \prime}\right)^{\star}=-P_{+} \chi_{S} Q_{y_{0}} \chi_{S}$. Therefore, equations (4.9)-(4.13) get replaced, respectively, by

$$
\begin{gathered}
{\left[\left(Q_{y_{0}} \chi_{S}\right)^{2}-\gamma_{2}^{\prime \prime}\right] \operatorname{Re} g_{2}^{\prime \prime}=Q_{y_{0}} \chi_{S} f_{2}-\gamma_{2}^{\prime \prime} h_{2},} \\
Q_{y_{0}}\left[\chi_{S} \varphi_{n}\right](x)=\frac{1}{\pi} \int_{S} \frac{(x-t) \varphi_{n}(t)}{(x-t)^{2}+y_{0}^{2}} d t=\lambda_{n} \varphi_{n}(x), \quad x \in S, \\
\operatorname{Re} g_{2}^{\prime \prime}(x)=\sum_{n=0}^{\infty} \frac{1}{\lambda_{n}^{2}-\gamma_{2}^{\prime \prime}}\left\langle Q_{y_{0}} \chi_{S} f_{2}-\gamma_{2}^{\prime \prime} h_{2}, \varphi_{n}\right\rangle_{L^{2}(S)} \varphi_{n}(x), \\
\sum_{n=0}^{\infty}\left[\left\langle\frac{1}{\lambda_{n}^{2}-\gamma_{2}^{\prime \prime}}\left(P_{y_{0}} \chi_{S} f_{2}-\gamma_{2}^{\prime \prime} h_{2}\right)-h_{2}, \varphi_{n}\right\rangle_{L^{2}(S)}\right]^{2}=M_{2}^{2}, \\
g_{2}^{\prime \prime}(x+i y)=\frac{1}{2}\left(P_{y}+i Q_{y}\right)\left[\chi_{S} \operatorname{Re} g_{2}^{\prime \prime}\right](x), \quad x+i y \in \Pi_{+},
\end{gathered}
$$

the logic remains precisely the same except that the existence of complete set of eigenfunctions $\varphi_{n}$ and eigenvalues $\lambda_{n}$ of $Q_{y_{0}} \chi_{S}$ is still guaranteed by the spectral theorem for compact normal operators (note that $\left.f \mapsto\left[Q_{y_{0}} \chi_{S} f\right]\right|_{S}$ is a compact anti-self-adjoint operator on $\left.L_{\mathbb{R}}^{2}(S)\right)$ [33, Thm 6.11.1].

\section{Conclusion}

Observe that in the particular situations where the function to be approximated already belongs to the approximant class, the above bounded extremal problems 0 and 1 coincide, in the absence of constraint, with recovery or extrapolation issues as in the works $[1,26,34]$, and with a constraint, to Loewner type interpolation issues as in [3]. It would be interesting to study similar interpolation / extrapolation issues related to Problem 2, for which the behaviour of the error (criterion) with respect to the constraint must be further analyzed as well. This should go together with numerical computation of the solutions.

Generalizations of Problem 2 to situations involving also the conjugate Poisson kernel are under study. This is also the case of some extensions of Problem 2 in the three-dimensional case, see [8] and [36, Part III]. Note that three-dimensional 
versions of Problem 0 have been considered in Hardy classes of harmonic gradients in spherical domains [5], while Hardy classes of the upper half-space are studied and used in [10].

Finally, observe that Problem 2 still makes sense in Hardy classes of generalized analytic (or pseudo-holomorphic) functions, as does Problem 0, see [9]. This deserves further study.

\section{References}

[1] L. Aizenberg, Carleman's formulas in complex analysis, Kluwer Acad. Pub., 1993.

[2] D. Alpay, An advanced complex analysis problem book, Birkhauser, 2015.

[3] D. Alpay, J. Leblond, Traces of Hardy functions and reproducing kernel Hilbert spaces, Archiv der Math., 64, 490-499, 1995.

[4] D. Alpay, L. Baratchart, J. Leblond, Some extremal problems linked with identification from partial frequency data, Proc. 10 Conf. Analyse Optimisation Systemes, Sophia-Antipolis, Springer-Verlag, LNCIS 185, 563-573, 1992.

[5] B. Atfeh, L. Baratchart, J. Leblond, J. R. Partington, Bounded extremal and Cauchy-Laplace problems on the sphere and shell, J. Fourier Analysis and Applications, 16 (2), 177-203, 2010.

[6] S. Axler, P. Bourdon, W. Ramey, Harmonic functions theory, Springer-Verlag, 2001.

[7] L. Baratchart, L. Bourgeois, J. Leblond, Uniqueness results for inverse Robin problems with bounded coefficients, J. Functional Analysis, 270 (7), 2508-2542, 2016.

[8] L. Baratchart, S. Chevillard, and J. Leblond, Silent and equivalent magnetic distributions on thin plates, To appear in Theta Series in Advanced Mathematics, http://hal.inria.fr/hal-01286117v2.

[9] L. Baratchart, Y. Fischer, J. Leblond, Dirichlet/Neumann problems and Hardy classes for the planar conductivity equation, Complex Variable \& Elliptic Equations, 59 (4), 504-538, 2014.

[10] L. Baratchart, D. Hardin, E. A. Lima, E. B. Saff, B. Weiss, Characterizing kernels of operators related to thin-plate magnetizations via generalizations of Hodge decompositions, Inverse Problems, 29 (1), 2013.

[11] L. Baratchart, J. Leblond, Hardy approximation to $L^{p}$ functions on subsets of the circle with $1 \leq p<\infty$, Constructive Approximation, 14, 41-56, 1998.

[12] L. Baratchart, J. Leblond, J. R. Partington, Hardy approximation to $L^{\infty}$ functions on subsets of the circle, Constructive Approximation, 12, 423-436, 1996.

[13] L. Baratchart, J. Leblond, J. R. Partington, N. Torkhani, Robust identification in the disc algebra from band-limited data, IEEE Trans. on Automatic Control, 42 (9), 1997.

[14] H. Brezis, Functional analysis, Sobolev spaces and partial differential equations, Universitext, Springer, 2011.

[15] S. Chaabane, I. Fellah, M. Jaoua, J. Leblond, Logarithmic stability estimates for a Robin coefficient in 2D Laplace inverse problems, Inverse problems, 20 (1), 49-57, 2004 . 
[16] I. Chalendar, J. Leblond, J. R. Partington, Approximation problems in some holomorphic spaces, with applications, Systems, Approximation, Singular Integral Operators, and Related Topics, Proceedings of IWOTA 2000, eds. A. A. Borichev, N. K. Nikolski, Integral Equations and Operator Theory, 129, 143-169, 2001.

[17] I. Chalendar, J. R. Partington, Constrained approximation and invariant subspaces. J. Math. Anal. Appl., 280, 176-187, 2003.

[18] I. Chalendar, J. R. Partington, Modern approaches to the invariant-subspace problem , Cambridge Tracts in Mathematics, 188, Cambridge University Press, 2011.

[19] P. L. Duren, Theory of $H^{p}$ spaces. Academic Press, New York, 1970.

[20] F. D. Gakhov, Boundary value problems, Dover Publications, 1990.

[21] J. B. Garnett, Bounded analytic functions, Academic Press, 1981.

[22] M. Jaoua, J. Leblond, M. Mahjoub, J. R. Partington, Robust numerical algorithms based on analytic approximation for the solution of inverse problems in annular domains, IMA J. of Applied Math., 74, 481-506, 2009.

[23] B. Jacob, J. Leblond, J.-P. Marmorat, J. R. Partington, A constrained approximation problem arising in parameter identification, Linear Algebra and its Applications, 351-352, 487-500, 2002.

[24] K. Hoffman, Banach spaces of analytic functions, Dover, 1988.

[25] P. Koosis, Introduction to $H_{p}$ spaces, Cambridge University Press, 1980.

[26] M. G. Krein, P. Y. Nudelman, Approximation of $L^{2}\left(\omega_{1}, \omega_{2}\right)$ functions by minimum energy transfer functions, Problemy Peredachi, 11 (2), 37-60, 1975.

[27] J. Leblond, J.-P. Marmorat, J. R. Partington, Solution of inverse diffusion problems by analytic approximation with real constraints, J. of Inverse and Ill-Posed Problems, 16 (1), 89-105, 2008.

[28] J. Leblond, J. R. Partington, Constrained approximation and interpolation in Hilbert function spaces, J. of Math. Anal. and Appl., 234 (2), 500-513, 1999.

[29] E. A. Lima, B. Weiss, L. Baratchart, D. Hardin, E. B. Saff, Fast inversion of magnetic field maps of unidirectional planar geological magnetization, Journal of Geophysical Research, 118 (6), 2013.

[30] J. Leblond, D. Ponomarev, Recovery of harmonic functions in planar domains from partial boundary data respecting internal values, Journal of Inverse and Ill-posed Problems, to appear, doi:10.1515/jiip-2015-0089.

[31] R. N. Mukherjee, Representations of $H^{2}$-functions on the real line, Boll. U.M.I. (4) 10, 666-671, 1974.

[32] N. I. Muskhelishvili, Singular Integral Equations, Dover Publications, 1992.

[33] A. W. Naylor, G. R. Sell, Linear Operator Theory in Engineering and Science, Springer, 1982.

[34] D. J. Patil, Representation of $H^{p}$ functions, Bull. Amer. Math. Soc., 78 (4), 1972.

[35] H. Pollard, The Poisson transform, Trans. Amer. Math. Soc. 78, 1955.

[36] D. Ponomarev, Some inverse problems with partial data, $\mathrm{PhD}$ thesis, Univ. Nice Sophia Antipolis, 2016.

[37] M. Smith, Approximation problems and operator theory in function spaces, $\mathrm{PhD}$ thesis, Univ. Leeds, 2002. 
[38] C. Standish, A generalization of the Poisson transform, Proc. Amer. Math. Soc., 6 (6), 942-949, 1955.

Juliette Leblond

Projet APICS, INRIA

2004 Route des Lucioles

06902 Sophia Antipolis Cedex,

France

e-mail: juliette.leblond@inria.fr

Dmitry Ponomarev

Laboratoire POEMS, ENSTA ParisTech

828 Boulevard des Maréchaux, 91120 Palaiseau

France

e-mail: dmitry.ponomarev@ensta-paristech.fr 